\title{
About a Problem for Loaded Parabolic-Hyperbolic Type Equation with Fractional Derivatives
}

\author{
Kishin B. Sadarangani' ${ }^{1}$ and Obidjon Kh. Abdullaev ${ }^{2}$ \\ ${ }^{1}$ Department of Functional Analysis and Integral Equations, University of Las Palmas de Gran Canaria, Campus de Tafira Baja, \\ Las Palmas, 35017 Gran Canaria, Spain \\ ${ }^{2}$ Faculty of Mechanics-Mathematics, Department of Differential Equations and Mathematical Physics, \\ National University of Uzbekistan, Universitetskaya 4, Almazar, 100125 Tashkent, Uzbekistan
}

Correspondence should be addressed to Obidjon Kh. Abdullaev; obidjon.mth@gmail.com

Received 12 February 2016; Revised 7 June 2016; Accepted 29 June 2016

Academic Editor: Nikolai A. Kudryashov

Copyright $\odot 2016$ K. B. Sadarangani and O. Kh. Abdullaev. This is an open access article distributed under the Creative Commons Attribution License, which permits unrestricted use, distribution, and reproduction in any medium, provided the original work is properly cited.

An existence and uniqueness of solution of local boundary value problem with discontinuous matching condition for the loaded parabolic-hyperbolic equation involving the Caputo fractional derivative and Riemann-Liouville integrals have been investigated. The uniqueness of solution is proved by the method of integral energy and the existence is proved by the method of integral equations. Let us note that, from this problem, the same problem follows with continuous gluing conditions (at $\lambda=1$ ); thus an existence theorem and uniqueness theorem will be correct and on this case.

\section{Introduction and Formulation of a Problem}

Development of the theory of the equations with fractional derivatives is stimulated with development of the theory of the integer order differential equations. About applications to physics, biology, mathematical modeling, and so forth, one can find works [1-3]. Notice works [4-7], devoted to the studying of BVPs for parabolic-hyperbolic equations, involving fractional derivatives. BVPs for the mixed type equations involving the Caputo and the Riemann-Liouville fractional differential operators were investigated in works $[8,9]$.

Note that with intensive research on problem of optimal control of the agroeconomic system, regulating the label of ground waters and soil moisture, it has become necessary to investigate a new class of equations called "loaded equations." It was given the most general definition of "loaded equations" and various loaded equations are classified in detail by Nakhushev [10]. After this work very interesting results on the theory of boundary value problems for the loaded equations parabolic, parabolic-hyperbolic, and elliptic-hyperbolic types were published; for example, see [11-13].

In this direction, some local and nonlocal problems for the loaded elliptic-hyperbolic type equations of the second and third order in double-connected domains were investigated (see [14-17]).

BVPs for the loaded mixed type equations with fractional derivative have not been investigated yet.

In the given work, we consider the following equation:

$$
0= \begin{cases}u_{x x}-{ }_{C} D_{o y}^{\alpha} u+p(x) \int_{x}^{1}(t-x)^{\beta-1} u(t, 0) d t, & \text { at } y>0 \\ u_{x x}-u_{y y}+q(x+y) \int_{x+y}^{1}(t-x-y)^{\gamma-1} u(t, 0) d t, & \text { at } y<0,\end{cases}
$$


with the following operation [18]:

$$
{ }_{C} D_{o y}^{\alpha} f=\frac{1}{\Gamma(1-\alpha)} \int_{0}^{y}(y-t)^{-\alpha} f^{\prime}(t) d t,
$$

where $0<\alpha, \beta, \gamma<1$.

Let $\Omega$ be domain, bounded with segments $\overline{A_{1} A_{2}}=$ $\{(x, y): x=1,0 \leq y \leq h\}, \overline{B_{1} B_{2}}=\{(x, y): x=0,0 \leq y \leq h\}$, and $\overline{B_{2} A_{2}}=\{(x, y): y=h, 0 \leq x \leq 1\}$ at $y>0$ and characteristics $A_{1} C: x-y=1 ; B_{1} C: x+y=0$ of $(1)$ at $y<0$, where $A_{1}(1 ; 0), A_{2}(1 ; h), B_{1}(0 ; 0), B_{2}(0 ; h), C(1 / 2 ;-1 / 2)$.

Let us enter designations

$$
\begin{aligned}
D_{x a}^{-\beta} f(x) & =\frac{1}{\Gamma(\beta)} \int_{x}^{a}(t-x)^{\beta-1} f(t) d t, \quad 0<\beta<1, \\
\Omega^{+} & =\Omega \cap(y>0), \\
\Omega^{-} & =\Omega \cap(y<0), \\
I_{1} & =\left\{x: \frac{1}{2}<x<1\right\}, \\
I_{2} & =\{y: 0<y<h\} .
\end{aligned}
$$

In the domain of $\Omega$ the following problem is investigated.

Problem 1. To find a solution $u(x, y)$ of (1) from the class of functions,

$$
\begin{aligned}
W & =\left\{u(x, y): u(x, y) \in C(\bar{\Omega}) \cap C^{2}\left(\Omega^{-}\right), u_{x x}\right. \\
& \left.\in C\left(\Omega^{+}\right),{ }_{C} D_{o y}^{\alpha} u \in C\left(\Omega^{+}\right)\right\},
\end{aligned}
$$

satisfying boundary conditions

$$
\begin{array}{cl}
\left.u(x, y)\right|_{A_{1} A_{2}}=\varphi(y), & y \in \bar{I}_{2}, \\
\left.u(x, y)\right|_{B_{1} B_{2}}=\psi(y), & y \in \bar{I}_{2}, \\
\left.u(x, y)\right|_{A_{1} C}=\omega(x), & x \in \overline{I_{1}},
\end{array}
$$

and gluing condition

$$
\lim _{y \rightarrow+0} y^{1-\alpha} u_{y}(x, y)=\lambda u_{y}(x,-0), \quad(x, 0) \in A_{1} B_{1},
$$

where $\varphi(y), \psi(y)$, and $\omega(x)$ are given functions, $\lambda=$ const $(\lambda \neq 0)$, and besides $\omega(1)=\varphi(0)$.

\section{The Uniqueness of Solution of Problem 1}

It is known that (1) at $y \leq 0$ on the characteristics coordinates $\xi=x+y$ and $\eta=x-y$ totally looks like

$$
u_{\xi \eta}=-\frac{q(\xi)}{4} \int_{\xi}^{1}(t-\xi)^{\gamma-1} u(t, 0) d t .
$$

Let us enter designations $u(x, 0)=\tau(x), 0 \leq x \leq 1$; $u_{y}(x,-0)=v^{-}(x), 0<x<1$

$$
\lim _{y \rightarrow+0} y^{1-\alpha} u_{y}(x, y)=v^{+}(x), \quad 0<x<1 .
$$

It is known that solution of the Cauchy problem for (1) in the domain of $\Omega^{-}$can be represented as follows:

$$
\begin{aligned}
u(x, y) & \\
= & \frac{\tau(x+y)+\tau(x-y)}{2}-\frac{1}{2} \int_{x+y}^{x-y} \nu^{-}(t) d t \\
& -\frac{1}{4} \int_{x+y}^{x-y} q(\xi) d \xi \int_{\xi}^{x-y} d \eta \int_{\xi}^{1}(t-\xi)^{\gamma-1} \tau(t) d t .
\end{aligned}
$$

After using condition (8) and taking (3) into account from (12) we will get

$$
\begin{aligned}
\nu^{-}(x)= & \frac{x-1}{2} \Gamma(\gamma) q(x) D_{x 1}^{-\gamma} \tau(x)-\tau^{\prime}(x) \\
& +\omega^{\prime}\left(\frac{x+1}{2}\right) .
\end{aligned}
$$

Considering designations and gluing condition (9) we have

$$
\nu^{+}(x)=\lambda \nu^{-}(x) .
$$

Further from (1) at $y \rightarrow+0$ taking into account (2), (14), and

$$
\lim _{y \rightarrow 0} D_{0 y}^{\alpha-1} f(y)=\Gamma(\alpha) \lim _{y \rightarrow 0} y^{1-\alpha} f(y),
$$

we get [8]

$$
\tau^{\prime \prime}(x)-\lambda \Gamma(\alpha) \nu^{-}(x)+\Gamma(\beta) p(x) D_{x 1}^{-\beta} \tau(x)=0 .
$$

Theorem 2. Satisfying conditions

$$
\begin{aligned}
p(0) & \leq 0, \\
p^{\prime}(x) & \leq 0 ; \\
\lambda q(0) & \leq 0, \\
\lambda((x-1) q(x))^{\prime} & \geq 0,
\end{aligned}
$$

the solution $u(x, y)$ of Problem 1 is unique.

Proof. It is known that if homogeneous problem has only trivial solution, then we can state that original problem has unique solution. For this aim we assume that Problem 1 has two solutions; then denoting difference of these solutions as $u(x, y)$ we will get appropriate homogenous problem.

We multiply (16) to $\tau(x)$ and integrated it from 0 to 1 :

$$
\begin{gathered}
\int_{0}^{1} \tau^{\prime \prime}(x) \tau(x) d x-\lambda \Gamma(\alpha) \int_{0}^{1} \tau(x) \nu^{-}(x) d x \\
+\Gamma(\beta) \int_{0}^{1} \tau(x) p(x) D_{x 1}^{-\beta} \tau(x) d x=0 .
\end{gathered}
$$

We will investigate the integral

$$
\begin{aligned}
I= & \lambda \Gamma(\alpha) \int_{0}^{1} \tau(x) \nu^{-}(x) d x \\
& -\Gamma(\beta) \int_{0}^{1} \tau(x) p(x) D_{x 1}^{-\beta} \tau(x) d x .
\end{aligned}
$$


Taking (13) into account, $\omega(x)=0$, we get

$$
\begin{aligned}
I= & \frac{\lambda \Gamma(\alpha) \Gamma(\gamma)}{2} \int_{0}^{1} \tau(x)(x-1) q(x) D_{x 1}^{-\gamma} \tau(x) d x \\
& -\lambda \Gamma(\alpha) \int_{0}^{1} \tau(x) \tau^{\prime}(x) d x-\Gamma(\beta) \\
& \cdot \int_{0}^{1} \tau(x) p(x) D_{x 1}^{-\beta} \tau(x) d x=\frac{\lambda \Gamma(\alpha)}{2} \\
& \cdot \int_{0}^{1} q(x) \tau(x)(1-x) d x \int_{x}^{1}(t-x)^{\gamma-1} \tau(t) d t \\
& -\frac{\lambda \Gamma(\alpha)}{2} \int_{0}^{1} d\left(\tau^{2}(x)\right)-\int_{0}^{1} \tau(x) p(x) d x \\
& \cdot \int_{x}^{1}(t-x)^{\beta-1} \tau(t) d t .
\end{aligned}
$$

Considering $\tau(1)=0, \tau(0)=0$ (deduced from conditions (6) and (7) in homogeneous case) and on a base of the formula (see $[19$, p. 188]),

$$
\begin{aligned}
& |x-t|^{-\gamma} \\
& =\frac{1}{\Gamma(\gamma) \cos (\pi \gamma / 2)} \int_{0}^{\infty} z^{\gamma-1} \cos [z(x-t)] d z, \\
& 0<\gamma<1 .
\end{aligned}
$$

After some simplifications from (20) we will get

$$
\begin{aligned}
I= & \frac{\lambda \Gamma(\alpha) q(0)}{4 \Gamma(1-\gamma) \sin (\pi \gamma / 2)} \\
& \cdot \int_{0}^{\infty} z^{-\gamma}\left[\left(\int_{0}^{1} \tau(t) \cos z t d t\right)^{2}\right. \\
& \left.+\left(\int_{0}^{1} \tau(t) \sin z t d t\right)^{2}\right] d z \\
& +\frac{\lambda \Gamma(\alpha)}{4 \Gamma(1-\gamma) \sin (\pi \gamma / 2)} \int_{0}^{\infty} z^{-\gamma} d z \\
& \cdot \int_{0}^{1} \frac{\partial}{\partial x}[(x-1) q(x)]\left[\left(\int_{x}^{1} \tau(t) \cos z t d t\right)^{2}\right. \\
& \left.+\left(\int_{x}^{1} \tau(t) \sin z t d t\right)^{2}\right] d x \\
& -\frac{p(0)}{2 \Gamma(1-\beta) \sin (\pi \beta / 2)} \\
& \cdot \int_{0}^{\infty} z^{-\beta}\left[\left(\int_{0}^{1} \tau(t) \cos z t d t\right)^{2}\right.
\end{aligned}
$$

$$
\begin{aligned}
& \left.+\left(\int_{0}^{1} \tau(t) \sin z t d t\right)^{2}\right] d z \\
& -\frac{1}{2 \Gamma(1-\beta) \sin (\pi \beta / 2)} \int_{0}^{\infty} z^{-\beta} d z \int_{0}^{1} \frac{\partial}{\partial x}[p(x)] \\
& \cdot\left[\left(\int_{x}^{1} \tau(t) \cos z t d t\right)^{2}\right. \\
& \left.+\left(\int_{x}^{1} \tau(t) \sin z t d t\right)^{2}\right] d x
\end{aligned}
$$

Thus, owing to (17) from (22) it is concluded that $\tau(x) \equiv 0$. Hence, based on the solution of the first boundary problem for (1) $[9,20]$ taking into account (6) and (7) we will get $u(x, y) \equiv 0$ in $\bar{\Omega}^{+}$. Further, from functional relations (13), taking into account $\tau(x) \equiv 0$, we get that $\nu^{-}(x) \equiv 0$. Consequently, based on the solution (12) we obtain $u(x, y) \equiv$ 0 in closed domain $\bar{\Omega}^{-}$.

\section{The Existence of Solution of Problem 1}

Theorem 3. Satisfying conditions (17) and

$$
\begin{gathered}
\varphi(y), \psi(y) \in C\left(\overline{I_{2}}\right) \cap C^{1}\left(I_{2}\right), \\
\omega(x) \in C^{1}\left(\overline{I_{1}}\right) \cap C^{3}\left(I_{1}\right), \\
p(x) \in C\left(\overline{A_{1} B_{1}}\right) \cap C^{2}\left(A_{1} B_{1}\right), \\
q(x+y) \in C\left(\overline{\Omega^{-}}\right) \cap C^{2}\left(\Omega^{-}\right),
\end{gathered}
$$

the solution of the investigating problem exists.

Taking (13) into account from (16) we will obtain

$$
\begin{aligned}
\tau^{\prime \prime}(x) & +\frac{\lambda}{2} \Gamma(\alpha) \Gamma(\gamma)(1-x) q(x) D_{x 1}^{-\gamma} \tau(x) \\
& +\lambda \Gamma(\alpha) \tau^{\prime}(x) \\
= & \lambda \Gamma(\alpha) \omega^{\prime}\left(\frac{x+1}{2}\right)-\Gamma(\beta) p(x) D_{x 1}^{-\beta} \tau(x) .
\end{aligned}
$$

From here,

$$
\tau^{\prime \prime}(x)+\lambda \Gamma(\alpha) \tau^{\prime}(x)=f(x)
$$

where

$$
\begin{aligned}
f(x)= & \frac{\lambda}{2} \Gamma(\alpha) \Gamma(\gamma)(x-1) q(x) D_{x 1}^{-\gamma} \tau(x) \\
& +\lambda \Gamma(\alpha) \omega^{\prime}\left(\frac{x+1}{2}\right)-\Gamma(\beta) p(x) D_{x 1}^{-\beta} \tau(x) .
\end{aligned}
$$

Solution of (25) together with conditions

$$
\begin{gathered}
\tau(0)=\psi(0), \\
\tau(1)=\varphi(0)
\end{gathered}
$$


has a form

$$
\tau(x)=(1-x) \psi(0)+x \varphi(0)+\int_{0}^{1} G(x, t) f_{1}(t) d t,
$$

where $f_{1}(x)=f(x)+\lambda \Gamma(\alpha)[\psi(0)-\varphi(0)]$. One has

$$
\begin{aligned}
& G(x, t) \\
& \quad= \begin{cases}\frac{\left(e^{\lambda \Gamma(\alpha) x}-1\right)\left(e^{\lambda \Gamma(\alpha) t}-e^{\lambda \Gamma(\alpha)}\right)}{e^{\lambda \Gamma(\alpha) x}\left(e^{\lambda \Gamma(\alpha)}-1\right) \lambda \Gamma(\alpha)} ; & 0 \leq t \leq x \\
\frac{\left(e^{\lambda \Gamma(\alpha) t}-1\right)\left(e^{\lambda \Gamma(\alpha) t}-e^{\lambda \Gamma(\alpha)}\right)}{e^{\lambda \Gamma(\alpha) x}\left(e^{\lambda \Gamma(\alpha)}-1\right) \lambda \Gamma(\alpha)} ; & t \leq x \leq 1 .\end{cases}
\end{aligned}
$$

$G(x, t)$ is Green's functions of the boundary value problem for

(25) with conditions (27). Further, considering (26) and using (3) from (28) we will get

$$
\begin{aligned}
\tau(x)= & \frac{\lambda \Gamma(\alpha)}{2} \int_{0}^{x} \tau(t) d t \\
& \cdot \int_{0}^{t}(s-1)(t-s)^{\gamma-1} K_{1}(x, s) q(s) d s \\
& -\int_{0}^{x} \tau(t) d t \int_{0}^{t}(t-s)^{\beta-1} K_{1}(x, s) p(s) d s \\
& +\frac{\lambda \Gamma(\alpha)}{2} \int_{x}^{1} \tau(t) d t \\
& \cdot \int_{0}^{x}(s-1)(t-s)^{\gamma-1} K_{1}(x, s) q(s) d s \\
& -\int_{x}^{1} \tau(t) d t \int_{0}^{x}(t-s)^{\beta-1} K_{1}(x, s) p(s) d s \\
& +\frac{\lambda \Gamma(\alpha)}{2} \int_{x}^{1} \tau(t) d t
\end{aligned}
$$

$$
\begin{aligned}
& \int_{t}^{x}(s-1)(t-s)^{\gamma-1} K_{2}(x, s) q(s) d s \\
- & \int_{x}^{1} \tau(t) d t \int_{x}^{t}(t-s)^{\beta-1} K_{2}(x, s) p(s) d s \\
+ & F(x),
\end{aligned}
$$

where

$$
\begin{aligned}
F(x)= & \lambda \Gamma(\alpha) \int_{0}^{1} G(x, t) \omega^{\prime}\left(\frac{t+x}{2}\right) d t \\
& -\lambda \Gamma(\alpha)(\psi(0)-\varphi(0)) \int_{0}^{1} G(x, t) d t, \\
K_{1}(x, t)= & \frac{\left(e^{\lambda \Gamma(\alpha) t}-1\right)\left(e^{\lambda \Gamma(\alpha) x}-e^{\lambda \Gamma(\alpha)}\right)}{e^{\lambda \Gamma(\alpha) x}\left(e^{\lambda \Gamma(\alpha)}-1\right) \lambda \Gamma(\alpha)} ; \\
K_{2}(x, t)= & \frac{\left(e^{\lambda \Gamma(\alpha) x}-1\right)\left(e^{\lambda \Gamma(\alpha) t}-e^{\lambda \Gamma(\alpha)}\right)}{e^{\lambda \Gamma(\alpha) x}\left(e^{\lambda \Gamma(\alpha)}-1\right) \lambda \Gamma(\alpha)} .
\end{aligned}
$$

Folding separately the integrals with limits $\int_{0}^{x}$ and $\int_{x}^{1}$ we rewrite integral equation (30) as follows:

$$
\tau(x)=\int_{0}^{1} K(x, t) \tau(t) d t+F(x)
$$

Here

$$
\begin{aligned}
K(x, t) \\
= \begin{cases}\int_{0}^{t} K_{1}(x, s)\left[\frac{\lambda \Gamma(\alpha)}{2}(1-s)(t-s)^{\gamma-1} q(s)-(t-s)^{\beta-1} p(s)\right] d s ; & 0 \leq t \leq x \\
\int_{0}^{x} K_{1}(x, s)\left[\frac{\lambda \Gamma(\alpha)}{2}(s-1)(t-s)^{\gamma-1} q(s)-(t-s)^{\beta-1} p(s)\right] d s+\int_{x}^{t} K_{2}(x, s)\left[\frac{\lambda \Gamma(\alpha)}{2}(s-1)(t-s)^{\gamma-1} q(s)-(t-s)^{\beta-1} p(s)\right] d s ; & x \leq t \leq 1 .\end{cases}
\end{aligned}
$$

For better understanding, we present the statement of equivalence.

Remark 4. Satisfying all conditions of Theorem 3, the existence and the uniqueness of solutions of Problem 1 in the class of $W$ are equivalent to the unique solvability of the Fredholm type integral equation (32) in the class of $C^{1}[0,1] \cap C^{2}(0,1)$.

Now we need to investigate the integral equation (32); for this aim we will estimate the kernel and the right side.
Owing to class (23) of the given functions and after some evaluations from (33) we will conclude that

$$
\begin{aligned}
& \left|K_{1}(x, t)\right|=\frac{\left(e^{\lambda \Gamma(\alpha) t}-1\right)}{\left(e^{\lambda \Gamma(\alpha)}-1\right) \lambda \Gamma(\alpha)}\left|1-e^{\lambda \Gamma(\alpha)(1-x)}\right| \\
& \leq \frac{\left|1-e^{\lambda \Gamma(\alpha)}\right|}{\lambda \Gamma(\alpha)}=\text { const }<\infty \quad \text { at } 0 \leq t \leq x, \\
& |K(x, t)|=\mid \int_{0}^{t} K_{1}(x, s)\left[\frac{\lambda \Gamma(\alpha)}{2}(1-s)(t-s)^{\gamma-1} q(s)\right.
\end{aligned}
$$




$$
\begin{aligned}
& \left.-(t-s)^{\beta-1} p(s)\right] d s \mid \leq \text { const } \mid \int_{0}^{t}(t \\
& -s)^{\sigma-1} d s \mid \leq \text { const } \cdot t^{\sigma} \leq \mathrm{const} \\
& \text { at } 0 \leq t \leq x \text {, where } \sigma=\min \{\gamma, \beta\} .
\end{aligned}
$$

Accordingly we can get $|K(x, t)| \leq$ const at $x \leq t \leq 1$.

Taking into account $\omega(x) \in C^{1}\left(\overline{I_{1}}\right) \cap C^{3}\left(I_{1}\right)$ and $|G(x, t)| \leq$ const and $\left|G_{x}(x, t)\right| \leq$ const from $F(x)=\lambda \Gamma(\alpha) \int_{0}^{1} G(x$, $t) \omega^{\prime}((t+x) / 2) d t-\lambda \Gamma(\alpha)(\psi(0)-\varphi(0)) \int_{0}^{1} G(x, t) d t$ we will get $|F(x)| \leq$ const and $\left|F^{\prime}(x)\right| \leq$ const. Hence on the base of theory of Fredholm integral equations of the second kind the integral equation (32) is solvability and we can write a solution of this equation via resolvent-kernel:

$$
\tau(x)=F(x)-\int_{0}^{1} \Re(x, t) F(t) d t
$$

where $\mathfrak{R}(x, t)$ is the resolvent-kernel of $K(x, t)$.

We will find unknown functions $\nu^{-}(x)$ and $\nu^{+}(x)$ accordingly from (13) and (14):

$$
\begin{aligned}
\nu^{-}(x) & \\
= & \frac{1-x}{2} q(x) \int_{x}^{1}(t-x)^{1-\gamma} d t \int_{0}^{1} \mathfrak{R}(t, s) F(s) d s \\
& -\frac{1-x}{2} q(x) \int_{x}^{1}(t-x)^{1-\gamma} F(t) d t-F^{\prime}(x) \\
& +\int_{0}^{1} \frac{\partial \mathfrak{R}(x, t)}{\partial x} F(t) d t+\omega^{\prime}\left(\frac{x+1}{2}\right),
\end{aligned}
$$

and $\nu^{+}(x)=\lambda \nu^{-}(x)$.

We write solution of Problem 1 in the domain $\Omega^{+}$as follows $[18,21]$ :

$$
\begin{aligned}
u(x, y)= & \int_{0}^{y} G_{\xi}(x, y, 0, \eta) \psi(\eta) d \eta \\
& -\int_{0}^{y} G_{\xi}(x, y, 1, \eta) \varphi(\eta) d \eta \\
& +\int_{0}^{1} G_{0}(x-\xi, y) \tau(\xi) d \xi \\
& -\int_{0}^{y} \int_{0}^{1} G(x, y, 0, \eta) p(\xi) d \xi d \eta \\
& \cdot \int_{\xi}^{1}(t-\xi)^{\beta-1} \tau(t) d t .
\end{aligned}
$$

$$
\begin{aligned}
& \text { Here } G_{0}(x-\xi, y)=(1 / \Gamma(1-\alpha)) \int_{0}^{y} \eta^{-\alpha} G(x, y, \xi, \eta) d \eta \\
& G(x, y, \xi, \eta)=\frac{(y-\eta)^{\alpha / 2-1}}{2} \\
& \cdot \sum_{n=-\infty}^{\infty}\left[e_{1, \alpha / 2}^{1, \alpha / 2}\left(-\frac{|x-\xi+2 n|}{(y-\eta)^{\alpha / 2}}\right)\right. \\
& \left.-e_{1, \alpha / 2}^{1, \alpha / 2}\left(-\frac{|x+\xi+2 n|}{(y-\eta)^{\alpha / 2}}\right)\right]
\end{aligned}
$$

is Green's function of the first boundary problem for (1) with the Riemann-Liouville fractional differential operator (note that it is true for the Caputo operator too) (see [20]), and

$$
e_{1, \delta}^{1, \delta}(z)=\sum_{n=0}^{\infty} \frac{z^{n}}{n ! \Gamma(\delta-\delta n)}
$$

is the Wright type function [18].

Solution of Problem 1 in the domain $\Omega^{-}$will be found by formula (12). Hence, Theorem 3 is proved.

\section{Competing Interests}

The authors declare that they have no competing interests.

\section{References}

[1] F. Mainardi, "Fractional calculus: some basic problem in continuum and statistical mechanics," in Fractal and Fractional Calculus in Continuum Mechanics, A. Carpinteri and F. Mainardi, Eds., pp. 291-948, Sprienger, Vienna, Austria, 1997.

[2] A. I. Saichev and G. M. Zaslavsky, "Fractional kinetic equations: solutions and applications," Chaos, vol. 7, no. 4, pp. 753-764, 1997.

[3] W. Wyss, "The fractional diffusion equation," Journal of Mathematical Physics, vol. 27, no. 11, pp. 2782-2785, 1986.

[4] A. A. Kilbas and O. A. Repin, "An analog of the Tricomi problem for a mixed type equation with a partial fractional derivative," Fractional Calculus \& Applied Analysis, vol. 13, no. 1, pp. 69-84, 2010.

[5] V. A. Nakhusheva, "Boundary problems for mixed type heat equation," Doklady AMAN, vol. 12, no. 2, pp. 39-44, 2010 (Russian).

[6] E. Y. Arlanova, "A problem with a shift for the mixed type equation with the generalized operators of fractional integration and differentiation in a boundary condition," Vestnik Samarsk Gosudarstvennogo Universiteta, vol. 6, no. 65, pp. 396-406, 2008 (Russian).

[7] A. A. Kilbas and O. A. Repin, "An analog of the BitsadzeSamarskii problem for a mixed type equation with a fractional derivative," Differentsial'nye Uravneniya, vol. 39, no. 5, pp. 638719,2003 , Translation in Journal of Difference Equations and Applications, vol. 39, no. 5, pp. 674-680, 2003.

[8] B. J. Kadirkulov, "Boundary problems for mixed parabolichyperbolic equations with two lines of changing type and fractional derivative," Electronic Journal of Differential Equations, vol. 2014, no. 57, pp. 1-7, 2014. 
[9] E. T. Karimov and J. S. Akhatov, "A boundary problem with integral gluing condition for a parabolic-hyperbolic equation involving the Caputo fractional derivative," Electronic Journal of Differential Equations, vol. 2014, no. 14, pp. 1-6, 2014.

[10] A. M. Nakhushev, The Loaded Equations and Their Applications, M. Nauka, 2012.

[11] V. A. Eleev, "About some boundary value problems for mixed type loaded equations of the second and third equations," Differential Equations, vol. 30, no. 2, pp. 230-237, 1994.

[12] V. M. Kaziev, "On a Darboux problem for the one loaded integral-differential equations of the second order," Differential Equations, vol. 14, no. 1, pp. 181-184, 1978.

[13] I. N. Lanin, "Boundary value problems for the loaded hyperbolic-parabolic type equations of the third equations," Differential Equations, vol. 17, no. 1, pp. 97-106, 1981.

[14] O. Kh. Abdullaev, "Boundary value problem for a loaded equation elliptic-hyperbolic type in double connected domain," Journal Collection of Scientific Works of KRASEC, vol. 1, no. 8, 2014.

[15] O. Kh. Abdullaev, "About a method of research of the nonlocal problem for the loaded mixed type equation in doubleconnected domain," Bulletin of KRASEC: Physical \& Mathematical Sciences, vol. 9, no. 2, pp. 11-16, 2014.

[16] O. Kh. Abdullaev, "Non-local boundary value problem for the mixed type equations on the third order in double-connected domains," Journal of PDE, vol. 27, no. 4, pp. 283-292, 2014.

[17] O. Kh. Abdullaev, "Non-local problem for the loaded mixed type equation with the integrated operators," in Proceedings of the International Conference. Differential Equations and Mathematical Modelin, pp. 21-23, Ulan-Ude, Russia, 2015.

[18] A. V. Pskhu, "Solution of boundary value problems fractional diffusion equation by the green function method," Differential Equation, vol. 39, no. 10, pp. 1509-1513, 2003.

[19] M. M. Smirnov, Mixed Type Equations, Nauka, Moscow, Russia, 2000.

[20] A. V. Pskhu, Partial Differential Equation of Fractional Order, Nauka, Moscow, Russia, 2005 (Russian).

[21] B. J. Kadirkulov and B. Kh. Turmetov, "On a generalization of the heat equation," $U z M J$, no. 3, pp. 40-46, 2006, http://uzmj .mathinst.uz/files/uzmj-2006_3.pdf. 


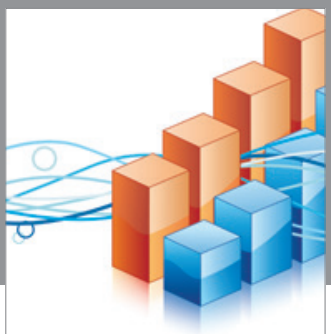

Advances in

Operations Research

vatem alat4

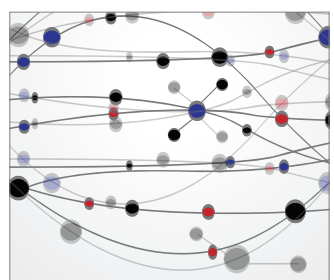

\section{The Scientific} World Journal
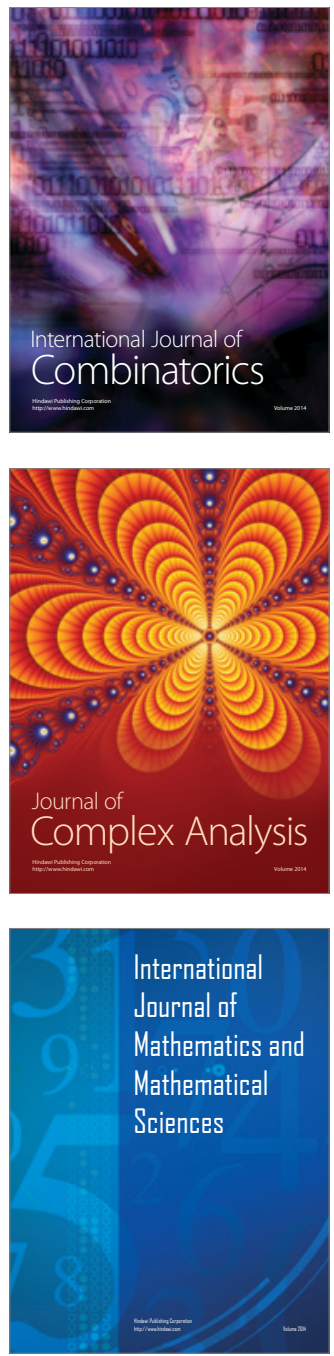
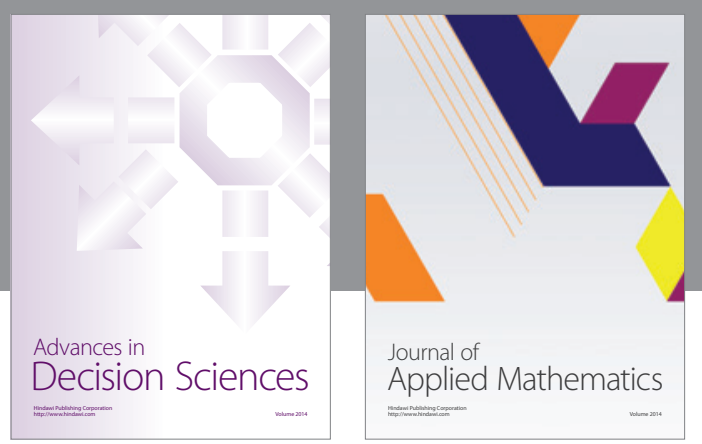

Algebra

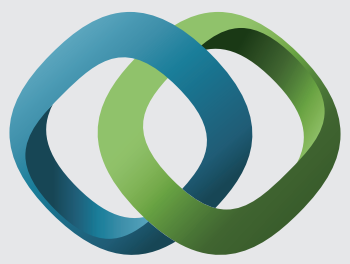

\section{Hindawi}

Submit your manuscripts at

http://www.hindawi.com
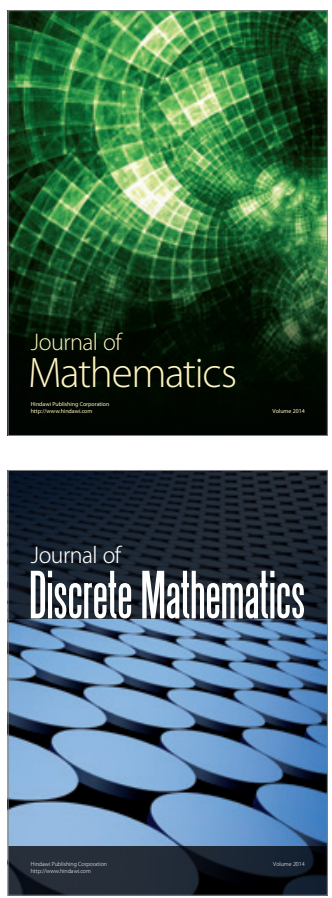

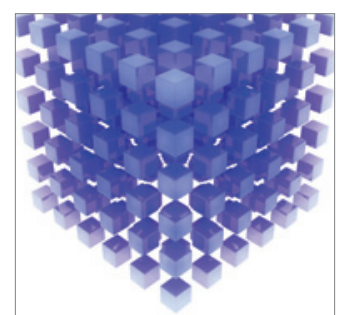

Mathematical Problems in Engineering
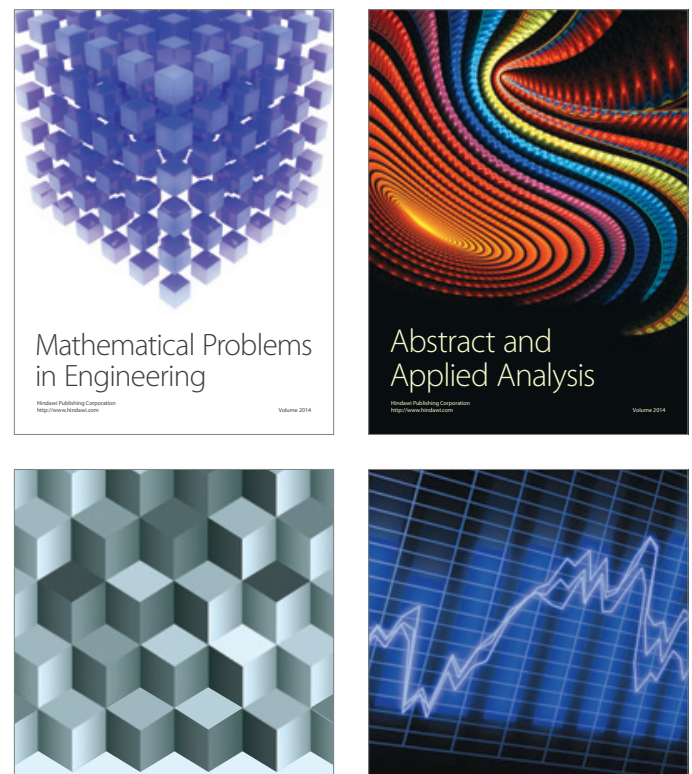

Journal of

Function Spaces

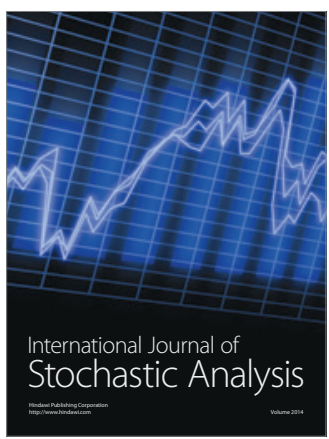

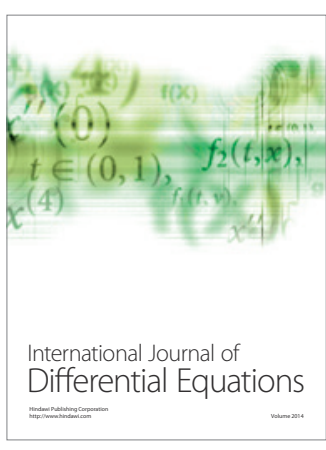
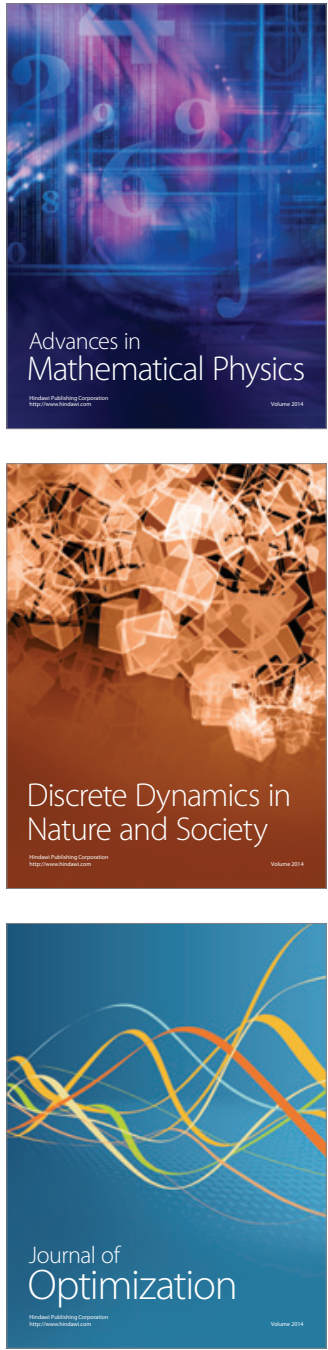